\title{
PLACE OF MAGNESIUM SULFATE IN CARDIOPULMONARY RESUSCITATION. A SYSTEMATIC REVIEW AND META-ANALYSIS
}

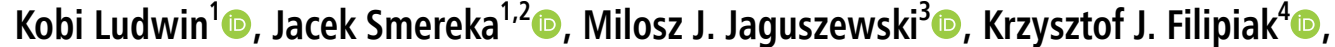 \\ Jerzy R. Ladny ${ }^{1,5}{ }^{\odot}$, Lukasz Szarpak ${ }^{1,6,7}{ }^{\infty}$, Sylwia Wozniak ${ }^{8}$, Togay Evrin ${ }^{9}$ \\ 'Polish Society of Disaster Medicine, Warsaw, Poland \\ ${ }^{2}$ Department of Emergency Medical Service, Wroclaw Medical University, Wroclaw, Poland \\ ${ }^{3}$ First Department of Cardiology, Medical University of Gdansk, Poland \\ ${ }^{4}$ First Chair and Department of Cardiology, Medical University of Warsaw, Poland \\ ${ }^{5}$ Department of Emergency Medicine, Medical University of Bialystok, Poland \\ ${ }^{6}$ Bialystok Oncology Center, Bialystok, Poland \\ ${ }^{7}$ Maria Sklodowska-Curie Medical Academy in Warsaw, Poland \\ ${ }^{8}$ Students Research Club, Maria Sklodowska-Curie Medical Academy in Warsaw, Poland \\ ${ }^{9}$ Department of Emergency Medicine, Ufuk University Medical Faculty, Dr Ridvan Ege Education and Research Hospital, Ankara, Turkey
}

\begin{abstract}
INTRODUCTION: Sudden cardiac arrest treatment is challenging, And the effectiveness of resuscitation procedures - especially in pre-hospital conditions - is low. The purpose of this meta-analysis is to investigate the effects of magnesium sulfate $\left(\mathrm{MgSO}_{4}\right)$ in cardiac arrest on the return of spontaneous circulation (ROSC) and survival to hospital discharge.
\end{abstract}

MATERIAL AND METHODS: We searched in MEDLINE, EMBASE, Scopus, and ClinicalTrials.gov, Web of Science up to May 25, 2020, and we conducted a systematic review and meta-analysis. We synthesized results by using mean differences, and odds ratios. The overall incidence and outcome of cardiac arrest were assessed using a random-effects meta-analysis.

RESULTS: A total of 5 eligible studies were included in this meta-analysis. Survival to discharge was higher in magnesium sulfate group compared to placebo group $(9,5 \%$ vs. $8.2 \%$ respectively; $\mathrm{OR}=1.17 ; 95 \% \mathrm{Cl}: 0.61$, $2.23 ; p=0.64)$. Higher survival rate to hospital admission was observed in the placebo group $-26.9 \% \mathrm{com}$ pared to the group where magnesium was administered $-25.7 \%(\mathrm{OR}=0.93 ; 95 \% \mathrm{Cl}: 0.59,1.47 ; \mathrm{p}=0.77$.

CONCLUSIONS: In conclusion, this meta-analysis indicates no statistically significant benefit of resuscitation with magnesium sulfate compared to the placebo. Thus, due to the low number of studies we recommend future randomized controlled trials to identify which anti-arrhythmic drug we should use on shock-refractory cardiac arrest.

KEY WORDS: magnesium sulfate, antiarrhythmic agents, cardiopulmonary resuscitation, advanced cardiovascular life support, meta-analysis, a systematic review

Disaster Emerg Med J 2020; 5(4)

\section{INTRODUCTION}

Magnesium is an important cofactor of several critical enzymes in the human body and is used both in pre-hospital care and in hospital wards. Assorting to the Deheinzelin et al. study incidence of hypo- 
magnesemia was reported in up to $65 \%$ in critically ill patients [1].

The therapeutic effect of magnesium sulfate is based on the antagonistic effect concerning calcium ions $\left(\mathrm{Ca}^{2+}\right)$ or blocking the release of catecholamines. This is due to the stabilizing effect of cell membranes by forming complexes with membrane phospholipids, which reduces the fluidity and permeability of cell membranes. $\mathrm{MgSO}_{4}$ also presents an effect on neuromuscular conduction by increasing the excitability threshold, which results in a decrease in the contractility of both striated and smooth muscles.

According to the characteristics of the drug $\mathrm{MgSO}_{4}$ is used in the treatment of magnesium deficiency: a) confirmed hypomagnesemia (e.g. In children with primary congenital hypomagnesemia, adults with malabsorption syndrome after persistent diarrhea, chronic alcoholism or prolonged parenteral nutrition); b) in the prevention and treatment of hypomagnesemia in patients who are parenterally fed exclusively; c) in the control and prevention of seizures in severe pre-eclampsia; $d$ ) in the control and prevention of recurrence of eclampsia; e) in torsade de pointes.

The use of $\mathrm{MgSO} 4$ requires medical personnel to monitor the patient as well as monitor blood magnesium levels. If magnesium concentration is $>6.2 \mathrm{mmol} / \mathrm{l}(15 \mathrm{mg} \%)$, bradycardia may occur, while if magnesium concentration is $>7.5 \mathrm{mmol} / \mathrm{l}$ (18 $\mathrm{mg} \%)$, conductivity disturbances and cardiac arrest due to hypermagnesemia may occur.

The advantages of administering magnesium in deficient states are known, but the benefits of routine administration of this element during a cardiac arrest have not been documented. However, some studies have shown that magnesium can be beneficial in resistant VF. Magnesium has well-known electrophysiological effects and normal concentrations are required to maintain normal cardiac conduction and rhythm. These actions of magnesium sulfate make it applicable in shock-resistant VF when hypomagnesemia is suspected; ventricular tachyarrhythmia when hypomagnesemia is suspected; torsades de pointes (polymorphic ventricular tachycardia) or digoxin poisoning. In stock-resistant $V F$, an initial dose of $2 \mathrm{~g}$ should be administered intravenously ( $4 \mathrm{ml}$ of $50 \%$ magnesium sulfate $=8 \mathrm{mmol}$ ) to the peripheral vascular bed in 1-2 minutes. It can be repeated after 10-15 minutes.

Therefore, we performed an updated meta-analysis of randomized controlled trials, addressing whether magnesium sulfate, compared with pla- cebo, improves survival outcome and good neurological outcome (Cerebral Performance Categories Scale, CPC 1,2) in adult patients with cardiac arrest. Besides, we analyzed outcomes of subgroups according to the location of arrest (out-of-hospital or in-hospital cardiac arrest). The primary objective was to determine whether magnesium sulfate results in better survival to discharge outcomes.

\section{MATERIAL AND METHODS}

This systematic review and meta-analysis adhered to the Preferred Reporting Items for Systematic Reviews and Meta-Analyses (PRISMA) guidelines [2] and is part of a larger systematic review that evaluated pharmacological effectiveness in cardiac arrest. Before starting the study, all authors agreed on the analysis methods and the inclusion and exclusion criteria to be applied. The protocol of this meta-analysis study has not been registered.

\section{Search strategy}

We searched Medline, PubMed, EMBASE, Scopus, Web of Science, and the Cochrane Database of Systematic Reviews from inception to 25 May 2020. The search terms were "magnesium" or " $\mathrm{MgSO}_{4}$ " and "cardiac arrest" or "IHCA" or "OHCA" or "ventricular fibrillation" or "VF" or "ventricular tachycardia" or "VT" or "CPR" or "cardiopulmonary resuscitation" or "sudden cardiac death" or "survival rate" or "mortality" or "return of spontaneous circulation". Only articles published in English were considered. Additionally, relevant clinical guidelines, systematic reviews, meta-analysis, and references of relevant publications were used to identify additional studies.

\section{Inclusion criteria and exclusion criteria}

Studies were included if they met the following eligibility criteria: a) evaluated adults aged at least 18 years old with cardiac arrest; b) compared magnesium sulfate with placebo; c) reported outcomes of interest; and d) were English language articles. We contacted the corresponding authors to obtain further information when these values could not be obtained from the reported data. Review articles, letters to the editor, conference papers, guidelines, editorials, and case reports were excluded.

\section{Data extraction}

Two independent reviewers (K. L. and J. S.) screened the titles and abstracts of all citations using pre- 
specified inclusion and exclusion criteria. Studies included by either reviewer were retrieved for fulltext screening. The same reviewers then screened the full-text version of eligible references. The following data were extracted from the studies: the first author's name, year of publication, a region of publication, number of patients, sex (male), age of patients. The rate of survival to hospital discharge was considered as the primary outcome. ROSC, survival with favorable neurological outcomes were also analyzed as outcome variables. Discrepancies between the reviewers were resolved through discussion. If consensus could not be reached, a third reviewer (L. S.) resolved the disagreement.

\section{Quality assessment}

Two authors (L. S. and J. S.) estimated the risk of bias. They evaluated the risk of bias of the included studies using Cochrane Collaboration "risk of bias" tool Review Manager software, version 5.3 (RevMan; Cochrane Collaboration, Oxford, UK) for randomized trials. According to the Higgins assessing the risk of bias guidelines [3] and previous meta-analysis the following domains were evaluated for RCTs: random sequence generation (selection bias), allocation concealment (selection bias), blinding of participants and personnel (performance bias), blinding of outcome assessment (detection bias), incomplete outcome data (attrition bias), selective reporting (reporting bias) and other bias [4]. Each was graded "yes", "no", or "unclear", which reflected a high risk of bias, low risk of bias, and uncertain bias, respectively. The review authors' judgments about each risk of bias item are provided in Supplementary digital file. The overall risk of bias for the study was rated 'low' if 7 or more domains were rated low, 'moderate' if 4 to 6 domains were rated low, and 'high' if 1 to 3 domains were rated low.

\section{Statistical analysis}

All statistical analyses were conducted using Review Manager version 5.3 (Cochrane Collaboration, Oxford, UK). A random-effects model was used to estimate the outcomes among cardiac arrest patients who received magnesium. The Mantel-Haenszel method was used to pool dichotomous data and to compute pooled odds ratios (ODs) with 95\% confidence intervals (Cls). The inverse variance method was used to pooled continuous data and to calcu- late weight mean differences with 95\% Cls. When the continuous outcome was reported in a study as median, range, and interquartile range, we estimated means and standard deviations using the formula described by Hozo et al. [5]. Statistical heterogeneity across studies was assessed with the $\mathrm{I}^{2}$ statistic, where values of $25 \%, 50 \%$, and $75 \%$ represented the cut-off points for low, moderate, and high levels of heterogeneity, respectively [6]. All statistical tests were two-sided and were considered when $\mathrm{p}<0.05$.

Subgroup analyses stratified by cardiac arrest place (in-hospital or out-of-hospital) were conducted to investigate potential sources of heterogeneity across subgroups. We performed sensitivity analyses by omitting one study at a time to assess the influence of any single study on the pooled survival rate estimates.

\section{RESULTS}

On May 25, 2020, 387 studies were retrieved from our literature search for review. After removing duplicate articles, we further excluded 289 articles based on titles and abstracts. A total of 22 articles were selected for further full-text assessment. After retrieving the full text for evaluation in detail, 17 articles were excluded because they did not meet our outcomes. Finally, a total of five studies were included in the present meta-analysis [7-11] (Fig. 1).

\section{Characteristics and quality of the studies included}

The characteristics of the 5 included studies are shown in Table 1. Three of those studies were conducted in the USA $[7,10,11]$, one in Australia [8], and one in the UK [9]. The year of publication ranged from 1997 to 2002. In total, 449 cardiac arrest patients were involved in this study. Five studies reported the incidence of return of spontaneous circulation [7-11], four the incidence of survival to hospital admission (or 24 hours survival) [7-9, 11], five studies survival to hospital discharge [7-11] and one study survival with favorable neurological outcome [10].

\section{Survival to discharge}

Survival to discharge was reported by five trials [7-11] and was higher in magnesium sulfate group compared to placebo group $(9,5 \%$ vs. $8.2 \%$ respectively; $\mathrm{OR}=1.17 ; 95 \% \mathrm{Cl}: 0.61,2.23 ; \mathrm{p}=0.64$ ) (Fig. 2). 


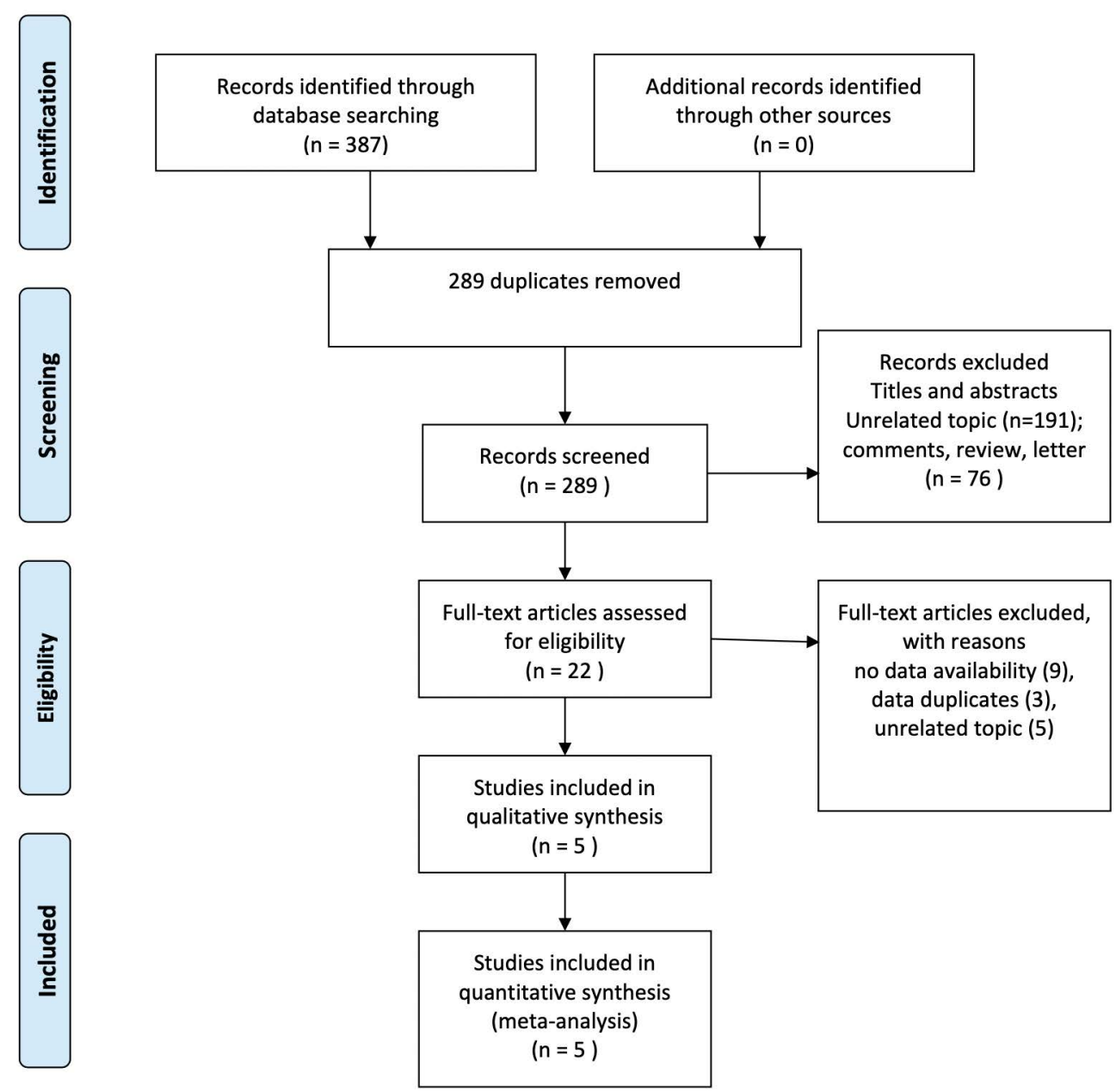

FIGURE 1. Flow diagram showing stages of database searching and study selection.

\begin{tabular}{|c|c|c|c|c|c|c|c|c|}
\hline \multirow{2}{*}{ Study } & \multirow{2}{*}{ Country } & \multirow{2}{*}{ Study design } & \multicolumn{3}{|c|}{$\mathrm{MgSO}_{4}$ group } & \multicolumn{3}{|c|}{ Control group } \\
\hline & & & $\mathrm{n}$ & Age & Sex, male & $\mathrm{n}$ & Age & Sex, male \\
\hline Allegra et al. 2001 & USA & Double-blinded RCT & 58 & $65(13)$ & $33(56.9 \%)$ & 58 & $65(14)$ & $27(46.6 \%)$ \\
\hline Fatovich et al. 1997 & Australia & Double-blinded RCT & 31 & $64(11.1)$ & $25(80.6 \%)$ & 36 & $65(12.8)$ & $31(86.1 \%)$ \\
\hline Hassan et al. 2002 & United Kingdom & Double-blinded RCT & 52 & $65(15)$ & $37(71.2 \%)$ & 53 & $67(12)$ & $37(55.2 \%)$ \\
\hline Miller et al. 1995 & USA & $\begin{array}{l}\text { Open label } \\
\text { randomized study }\end{array}$ & 23 & 72.3 & NR & 33 & 73.6 & NR \\
\hline Thel et al. 1997 & USA & $\begin{array}{c}\text { Open label } \\
\text { randomized study }\end{array}$ & 76 & $61.8(3.5)$ & $46(60.5 \%)$ & 80 & $67(2.7)$ & $43(53.8 \%)$ \\
\hline
\end{tabular}

NR — Not reported; RCT — Randomized controlled trial

In out-of-hospital cardiac arrest survival to hospital discharge for magnesium was $3.6 \%$ and was $1.5 \%$ higher than in the placebo group $(\mathrm{OR}=1.60$; $95 \% \mathrm{Cl}: 0.40,6.43 ; p=0.51)$. In in-hospital cardiac arrest group survival to hospital discharge in magnesium sulfate group and placebo group var- ied and amounted to $17.2 \%$ vs. $15.9 \%$ respectively $(\mathrm{OR}=1.07 ; 95 \% \mathrm{Cl}: 0.51,2.23 ; \mathrm{p}=0.86)$.

\section{Return of spontaneous circulation}

Five studies reported a return of spontaneous circulation [7-11]. In pooled analysis range of ROSC for 


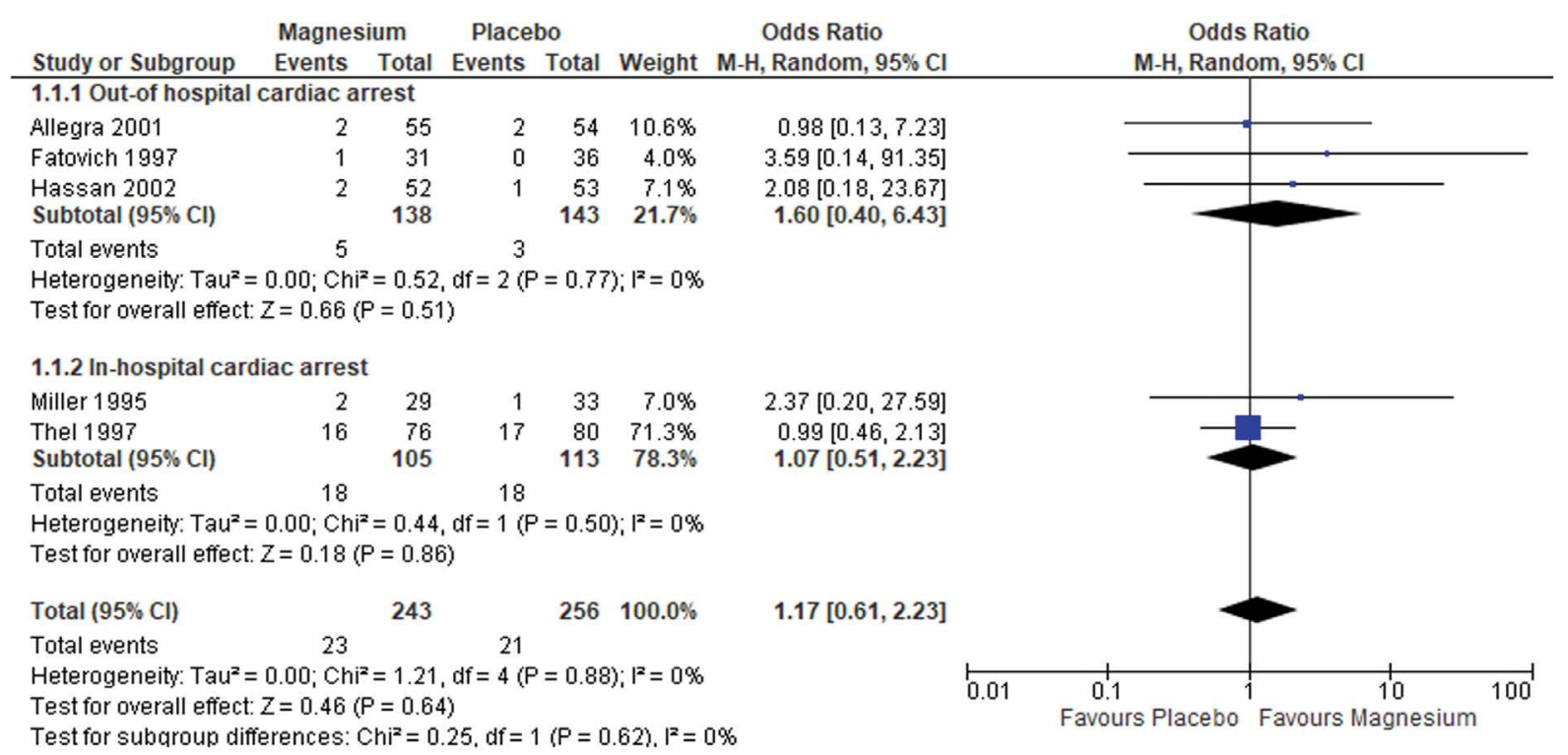

FIGURE 2. Forest plot of survival to discharge in magnesium vs. non-magnesium groups. The center of each square represents the odds ratio for individual trials, and the corresponding horizontal line stands for a $95 \%$ confidence interval. The diamonds represent pooled results.

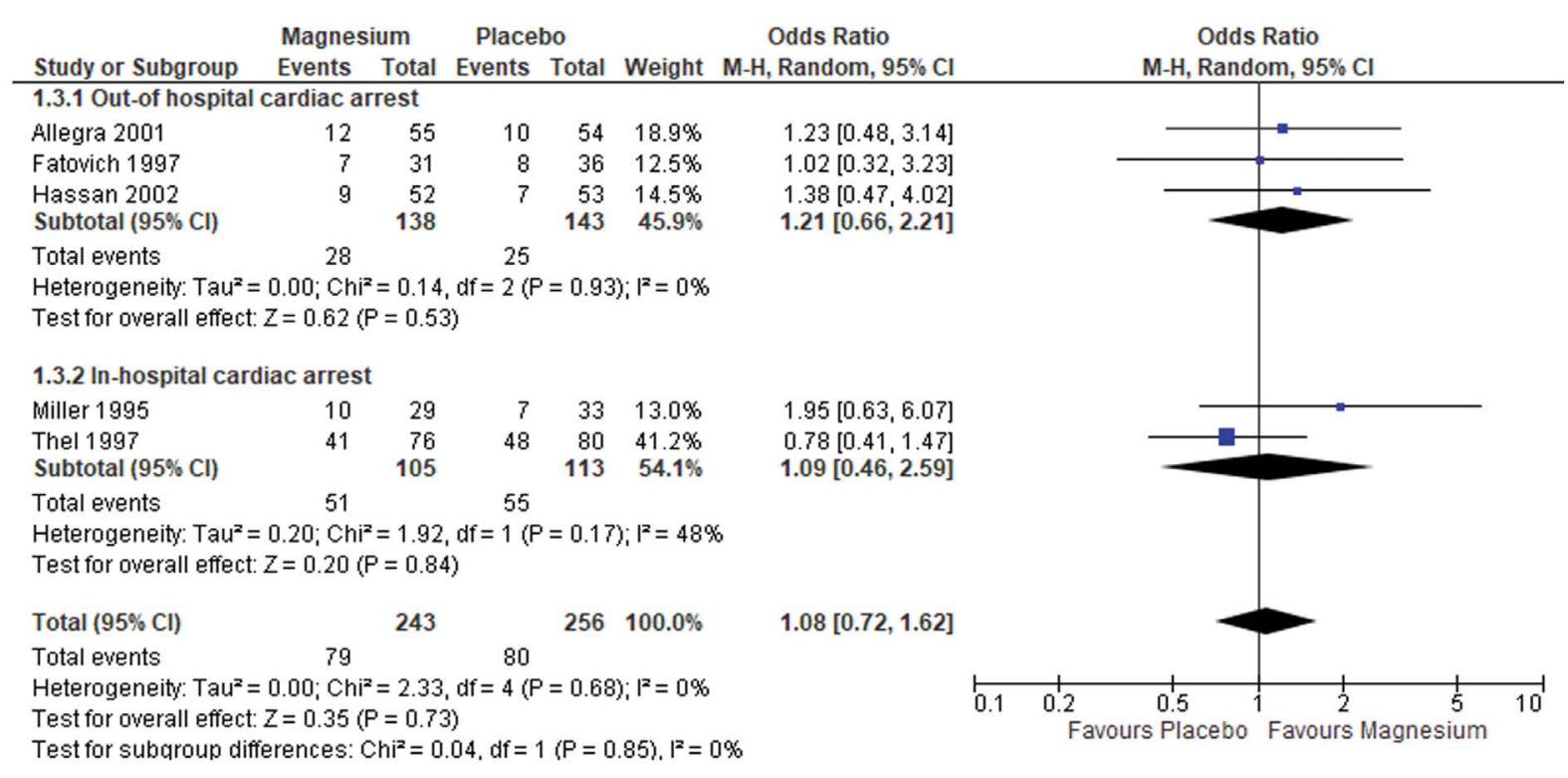

FIGURE 3. Forest plot of return of spontaneous circulation in magnesium vs. non-magnesium groups. The center of each square represents the odds ratio for individual trials, and the corresponding horizontal line stands for a $95 \%$ confidence interval. The diamonds represent pooled results.

magnesium was $32.5 \%$ and was slightly higher than for placebo $-31.3 \%(\mathrm{OR}=1.21 ; 95 \% \mathrm{Cl}$ : 0.66 , 2.21; $p=0.73$ ) (Fig. 3). Subgroup analysis showed that for out-of-hospital cardiac arrest the efficacy of ROSC was observed in $20.3 \%$ of cases in the magnesium group and $17.5 \%$ in the placebo group $(\mathrm{OR}=1.21 ; 95 \% \mathrm{Cl}: 0.66,2.21 ; \mathrm{p}=0.53)$. In in-hospital cardiac arrest the rate of ROSC was $48.6 \%$ vs. $48.7 \%$ respectively $(\mathrm{OR}=1.09 ; 95 \% \mathrm{Cl}: 0.46$, $2.59 ; p=0.84)$.

\section{Survival to hospital admission/24 hours}

Four studies reported survival to hospital admission or 24 hours survival $[7-9,11]$. Higher survival rate was observed in the placebo group - $26.9 \%$ compared to the group where magnesium was admin- 


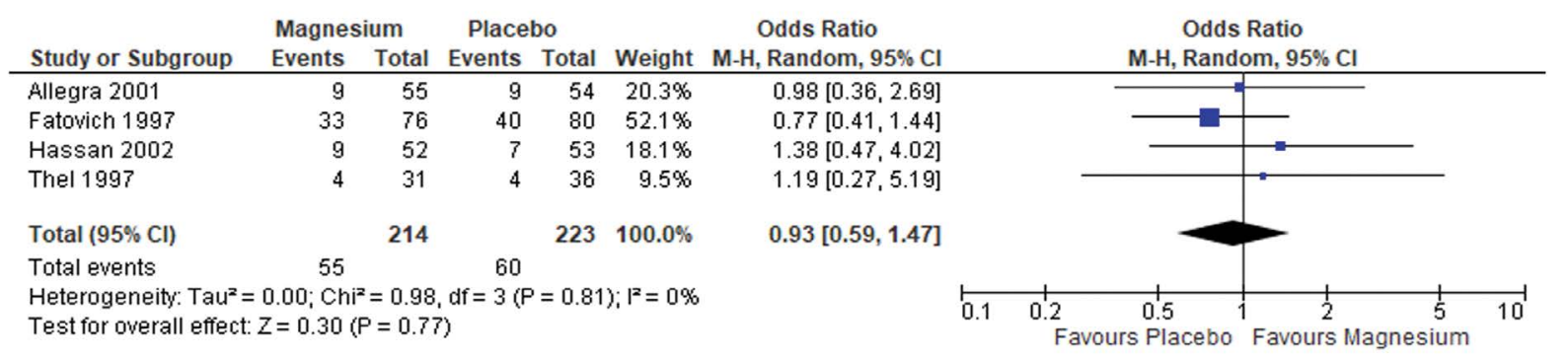

FIGURE 4. Forest plot of survival to hospital admission in magnesium vs. non-magnesium groups. The center of each square represents the odds ratio for individual trials, and the corresponding horizontal line stands for a $95 \%$ confidence interval. The diamonds represent pooled results.

istered $-25.7 \%(\mathrm{OR}=0.93 ; 95 \% \mathrm{Cl}: 0.59,1.47$; $\mathrm{p}=0.77$ ) (Fig. 4).

\section{Survival with favorable neurological outcome}

Only study by Miller et al. [10] reported survival to hospital discharge with favorable neurological outcome. This outcome was comparable between the groups magnesium sulfate vs. placebo (3.4\% vs. $3.0 \% ; \mathrm{OR}=1.14 ; 95 \% \mathrm{Cl}: 0.07,19.13$; $\mathrm{p}=0.93)$.

\section{DISCUSSION}

We conducted a systematic review and meta-analysis to evaluate the association magnesium sulfate with survival after out-of-hospital as well as in-hospital cardiac arrest.

Magnesium sulphate has a wide range of applications in emergency medicine. It is mainly used in ventricular fibrillation or shock-resistant tachycardia as well as in other life-threatening tachyarrhythmias, especially when they result from hypomagnesemia, in torsades de pointes $[12,13]$ or in situations where there is a suspicion of cardiac arrest due to poisoning with cardiac glycosides [14], in acute asthma $[15,16]$ or acute stroke $[17,18]$.

Magnesium itself is a component of many enzymatic systems that determine the production of energy in the muscles. It is also an antagonist of calcium ions causing a decrease in the systolic strength and heart rate while improving the systolic activity of the myocardium [18].

Magnesium sulfate is considered an antiarrhythmic drug. Currently, there is no unanimous approach to its use during cardiopulmonary resuscitation. In a trial by Fatovich et al. high dose of magnesium as first-line drug therapy for out-of-hospital cardiac arrest was not associated with significantly improved survival [8]. The Hassan et al study also reached similar conclusions [9].

A study performed by Upala et al. found a strong association between hypomagnesemia and increased mortality in ICU patients [20]. In turn, a study by Johnson et al. relating to the evaluation of the correlation between cord blood magnesium concentration and the effectiveness of resuscitation concerning neonatal resuscitation showed no relationship between cord blood $\mathrm{Mg}$ and delivery room resuscitation [21].

An increase in adrenaline levels during resuscitation may increase the release of magnesium, which creates a toning effect utilizing negative feedback resulting in the release of magnesium from the storage pool and thus lowering the adrenaline levels as a final result $[22,23]$. Magnesium ions, as already mentioned in the introduction, influence the activity of enzymes regulating carbohydrate metabolism and modulate the supply of glucose to nerve cells. Magnesium ions also have a stabilizing effect on the cell membranes of thrombocytes, thus reducing platelet aggregation [24, 25].

The present study has several potential limitations. We only these studies published in English were included in this meta-analysis and studies in other languages were omitted. Another limitation is the small number of studies on the effectiveness of magnesium sulfate during cardiopulmonary resuscitation.

\section{CONCLUSIONS}

In conclusion, this meta-analysis indicates no statistically significant benefit of resuscitation with magnesium sulfate compared to the placebo. Thus, due to the low number of studies we recommend future randomized controlled trials to identify which 
anti-arrhythmic drug we should use on shock-refractory cardiac arrest.

\section{Supplementary digital file}

Supplementary material related to this article can be found, in the online version, at: https://journals. viamedica.pl/disaster_and_emergency_medicine/article/view/DEMJ.a2020.0041\#supplementaryFiles

Funding sources: None.

Conflicts of interest: None.

Acknowledgments: Study supported by the ERC Research NET and Polish Society of Disaster Medicine.

\section{REFERENCES}

1. Deheinzelin D, Negri EM, Tucci MR, et al. Hypomagnesemia in critically ill cancer patients: a prospective study of predictive factors. Braz J Med Biol Res. 2000; 33(12): 1443-1448, doi: 10.1590/s0100879x2000001200007, indexed in Pubmed: 11105096.

2. Liberati A, Altman DG, Tetzlaff J, et al. The PRISMA statement for reporting systematic reviews and meta-analyses of studies that evaluate healthcare interventions: explanation and elaboration. BMJ. 2009; 339: b2700, doi: 10.1136/bmj.b2700, indexed in Pubmed: 19622552.

3. Savović J, Weeks L, Sterne JAC, et al. Cochrane Bias Methods Group, Cochrane Statistical Methods Group. The Cochrane Collaboration's tool for assessing risk of bias in randomised trials. BMJ. 2011; 343(1): d5928-85, doi: 10.1136/bmj.d5928, indexed in Pubmed: 22008217.

4. Szarpak L, Filipiak KJ, Mosteller L, et al. Survival, neurological and safety outcomes after out of hospital cardiac arrests treated by using prehospital therapeutic hypothermia: A systematic review and meta-analysis. Am J Emerg Med. 2020 [Epub ahead of print], doi: 10.1016/j.ajem.2020.02.019, indexed in Pubmed: 32088060.

5. Hozo SP, Djulbegovic B, Hozo I. Estimating the mean and variance from the median, range, and the size of a sample. BMC Med Res Methodol. 2005; 5: 13, doi: 10.1186/1471-2288-5-13, indexed in Pubmed: 15840177.

6. Higgins JPT, Thompson SG. Quantifying heterogeneity in a meta-analysis. Stat Med. 2002; 21(11): 1539-1558, doi: 10.1002/sim.1186, indexed in Pubmed: 12111919.

7. Allegra J, Lavery R, Cody R, et al. Magnesium sulfate in the treatment of refractory ventricular fibrillation in the prehospital setting. Resuscitation. 2001; 49(3): 245-249, doi: 10.1016/50300-9572(00)00375-0, indexed in Pubmed: 11719117.

8. Fatovich DM, Prentice DA, Dobb GJ. Magnesium in cardiac arrest (the magic trial). Resuscitation. 1997; 35(3): 237-241, doi: 10.1016/ s0300-9572(97)00062-2, indexed in Pubmed: 10203402.

9. Hassan TB, Jagger C, Barnett DB. A randomised trial to investigate the efficacy of magnesium sulphate for refractory ventricular fibrillation.
Emerg Med J. 2002; 19(1): 57-62, doi: 10.1136/emj.19.1.57, indexed in Pubmed: 11777881.

10. Miller B, Craddock L, Hoffenberg $S$, et al. Pilot study of intravenous magnesium sulfate in refractory cardiac arrest: safety data and recommendations for future studies. Resuscitation. 1995; 30(1): 3-14, doi: 10.1016/0300-9572(95)00868-t, indexed in Pubmed: 7481101.

11. Thel MC, Armstrong AL, McNulty SE, et al. Randomised trial of magnesium in in-hospital cardiac arrest. Duke Internal Medicine Housestaff. Lancet. 1997; 350(9087): 1272-1276, doi: 10.1016/ s0140-6736(97)05048-4, indexed in Pubmed: 9357406.

12. Thomas SHL, Behr ER. Pharmacological treatment of acquired QT prolongation and torsades de pointes. Br J Clin Pharmacol. 2016; 81(3): 420-427, doi: 10.1111/bcp.12726, indexed in Pubmed: 26183037.

13. Lazzerini PE, Bertolozzi I, Finizola F, et al. Proton Pump Inhibitors and Serum Magnesium Levels in Patients With Torsades de Pointes. Front Pharmacol. 2018; 9: 363, doi: 10.3389/fphar.2018.00363, indexed in Pubmed: 29731714.

14. Geiger H, Wanner C. Magnesium in disease. Clin Kidney J. 2012; 5(Suppl 1): i25-i38, doi: 10.1093/ndtplus/sfr165, indexed in Pubmed: 26069818.

15. Normansell R, Knightly R, Milan SJ, et al. Inhaled magnesium sulfate in the treatment of acute asthma. Cochrane Database Syst Rev. 2017; 11: CD003898-33, doi: 10.1002/14651858.CD003898.pub6, indexed in Pubmed: 29182799.

16. Griffiths $B$, Kew KM, Normansell $R$, et al. Intravenous magnesium sulfate for treating children with acute asthma in the emergency department. Cochrane Database Syst Rev. 2016; 4: CD011050-47, doi: 10.1002/14651858.CD011050.pub2, indexed in Pubmed: 27126744.

17. Bilotta F, Gelb AW, Stazi E, et al. Pharmacological perioperative brain neuroprotection: a qualitative review of randomized clinical trials. $\mathrm{Br}$ J Anaesth. 2013; 110 Suppl 1: i113-i120, doi: 10.1093/bja/aet059, indexed in Pubmed: 23562933.

18. Dorhout Mees SM, Rinkel GJE, Feigin VL, et al. Calcium antagonists for aneurysmal subarachnoid haemorrhage. Cochrane Database Syst Rev. 2007(3): CD000277, doi: 10.1002/14651858.CD000277.pub3, indexed in Pubmed: 17636626.

19. Dorhout Mees SM, Rinkel GJE, Feigin VL, et al. Calcium antagonists for aneurysmal subarachnoid haemorrhage. Cochrane Database Syst Rev. 2007(3): CD000277, doi: 10.1002/14651858.CD000277.pub3, indexed in Pubmed: 17636626.

20. Upala S, Jaruvongvanich V, Wijarnpreecha K, et al. Hypomagnesemia and mortality in patients admitted to intensive care unit: a systematic review and meta-analysis. QJM. 2016; 109(7): 453-459, doi: 10.1093/ qjmed/hcw048, indexed in Pubmed: 27016536.

21. Johnson LH, Mapp DC, Rouse DJ, et al. Eunice Kennedy Shriver National Institute of Child Health and Human Development Maternal-Fetal Medicine Units Network. Association of cord blood magnesium concentration and neonatal resuscitation. J Pediatr. 2012; 160(4): 573-577. e1, doi: 10.1016/j.jpeds.2011.09.016, indexed in Pubmed: 22056282.

22. James MF, Cork RC, Harlen GM, et al. Interactions of adrenaline and magnesium on the cardiovascular system of the baboon. Magnesium. 1988; 7(1): 37-43, indexed in Pubmed: 3379980. 
23. Mayer DB, Miletich DJ, Feld JM, et al. The effects of magnesium salts on the duration of epinephrine-induced ventricular tachyarrhythmias in anesthetized rats. Anesthesiology. 1989; 71(6): 923-928, doi: 10.1097/00000542-198912000-00016, indexed in Pubmed: 2589679.

24. Rysánek K, König J, Spánková $H$, et al. The effect of magnesium on the aggregation of human thrombocytes induced by adrena- line. Act Nerv Super (Praha). 1970; 12(3): 258-259, indexed in Pubmed: 5457447.

25. Brown CG, Griffith RF, Neely D, et al. The effect of intravenous magnesium administration on aortic, right atrial and coronary perfusion pressures during CPR in swine. Resuscitation. 1993; 26(1): 3-12, doi: 10.1016/0300-9572(93)90157-I, indexed in Pubmed: 8210728. 